\title{
CULTURA «PARA» EL TRABAJO. EL CAPITAL CULTURAL Y SU LÓGICA EN LAS ESTRATEGIAS LABORALES DE JÓVENES DE UN BARRIO POPULAR DE CÓRDOBA
}

GONZALO ASSUSA*

\begin{abstract}
RESUMEN
El diagnóstico de la problemática laboral para jóvenes está generalmente asociada a dos dimensiones: la primera, referida a las cuestiones vinculadas a la «cultura del trabajo» y su desestructuración tras años de desempleo masivo; y la segunda, referida a los problemas de calificación.

Sin embargo, limitados por enfoques «legitimistas», muchos de estos diagnósticos desconocen la existencia de lógicas específicamente populares de funcionamiento del capital cultural, de espacios diferenciales y de apuestas vinculadas a disposiciones singulares. El presente texto pretende discutir, desde la perspectiva de la cultura popular, la categoría de «capital cultural» aplicada a la comprensión de las estrategias laborales de jóvenes de un barrio popular de la ciudad de Córdoba.
\end{abstract}

\footnotetext{
PALABRAS CLAVE: ESTRATEGIAS LABORALES, CAPITAL CULTURAL, CULTURA POPULAR
}

* Argentino, Licenciado en Sociología por la Universidad Nacional de Villa María y Doctorando en Ciencias Antropológicas por la Universidad Nacional de Córdoba. Becario de postgrado de CONICET en IDHCIFFYH-UNC. Email: gon_assusa@ hotmail.com. 


\title{
CULTURA «PARA»O TRABALHO: O CAPITAL CULTURAL E SUA LÓGICA NAS ESTRATÉGIAS DE TRABALHO DE JOVENS DE UM BAIRRO POPULAR DE CORDOBA CAPITAL
}

\begin{abstract}
RESUMO
Geralmente, o diagnóstico dos problemas relativos ao trabalho para jovens está associado a duas dimensões: a primeira, refere-se a questões relacionadas com a «cultura de trabalho» e sua desintegração após anos de desemprego em massa; a segunda, refere-se a problemas de qualificação. No entanto, limitados pelas abordagens «legitimistas», muitos destes diagnósticos ignoram a existência de lógicas especificamente populares de funcionamento do capital cultural, de espaços diferenciais e de apostas vinculadas a disposições singulares. Este artigo discute — a partir da perspectiva da cultura popular-, a categoria de «capital cultural», aplicada à compreensão das estratégias de trabalho dos jovens de um bairro da cidade de Córdoba.

\author{
CAPITAL CULTURAL, CULTURA COPULAR
}

PALAVRAS CHAVE: ESTRATÉGIAS DE TRABALHO,

\section{CULTURE «FOR» WORK. \\ CULTURAL CAPITAL AND HIS LOGIC LABOR STRATEGIES TO YOUNG PEOPLE OF A POPULAR NEIGHBORHOOD IN CAPITAL CORDOBA}

\begin{abstract}
Diagnosis of youth employment problems generally associated with two dimensions: the first, referred to issues relating to the «work culture» and its disintegration after years of massive unemployment, and the second, based on the qualification problems.

However, limited by «loyalists» approaches, many of these diagnoses are unaware of the existence of specifically popular logics of functioning of the cultural capital, of differential spaces and of bets linked to singular dispositions. This paper discusses, from the perspective of popular culture, the category of «cultural capital» applied to the understanding of the business strategies of youth from a poor neighborhood of the city of Córdoba.
\end{abstract}

KEY WORDS: LABOR STRATEGIES, CULTURAL CAPITAL, POPULAR CULTURE 


\section{INTRODUCCIÓN ${ }^{1}$}

CONSTITUYE UN LUGAR común en los diagnósticos sobre los problemas de empleo el señalamiento de que la titulación escolar media es uno de los factores fundamentales para la explicación de las causas del desempleo, tanto como para el subempleo y los empleos precarios. «En un contexto caracterizado por un mercado de trabajo cada vez más inestable y precario, la escuela aparece como condición imprescindible para acceder al trabajo. A su vez, el trabajo es el medio para tener una «vida digna», para «ser alguien en la vida» [...] En definitiva, el trabajo y la escuela siguen siendo percibidos como dos medios imprescindibles para la integración social» (Cafiero, 2007).

En el marco de ese diagnóstico, buena parte de las políticas de empleo para jóvenes en la postconvertibilidad Argentina (Neffa, Brown y López, 2012) incluyen entre sus prestaciones la promoción - a través de una serie de incentivos, ayudas y exigencias- para la finalización de los estudios formales. Sin embargo, los supuestos teórico-metodológicos que orientan estas definiciones y tecnologías de regulación sobre el «mundo del trabajo» tienden a generar algunas homogeneizaciones y sustantivaciones que es preciso discutir para llegar a una comprensión más acabada de la cuestión. Las distintas formas de saberes, conocimientos y credenciales educativas acumuladas e invertidas en búsquedas e inserciones laborales no ocupan los mismos lugares en las distintas clases sociales, fracciones de clase o fragmentos del mercado de trabajo. Es necesario reconocer las condiciones y trayectorias diferenciales que habilitan procesos de valorización de los recursos culturales sumamente desiguales en distintos grupos de la sociedad. ${ }^{2}$

1 Agradezco la lectura, críticas, observaciones y correcciones sobre el texto de mis compañeros Nicolás Cabrera, Ivana Bartolozzi y María Laura Freyre, y de mi directora Alicia Gutiérrez.

2 La investigación en la cual el presente estudio se inserta, aún en curso, intenta reconstruir las trayectorias y prácticas laborales de jóvenes de un barrio popular del sur de la ciudad de Córdoba Capital. En lo que va del trabajo de campo, se realizaron entrevistas a jóvenes de un programa de empleo (Jóvenes con más y mejor trabajo, en adelante PJM y MT) del Ministerio de Trabajo, Empleo y Seguridad Social de la Nación (MTESS). También se hicieron entrevistas a agentes estatales dedicados a la cuestión del empleo, a referentes barriales y a docentes de establecimientos educativos de la zona. Por último, se registraron observaciones 
Considerando lo anterior, aquí analizaremos críticamente el diagnóstico realizado sobre las «posibilidades de éxito» en las búsquedas laborales de los jóvenes en relación a la distribución del capital cultural. El objetivo del trabajo será, entonces, evaluar, en el estudio de caso que investigamos, y en relación con los procesos estructurales que representa y en los que se inserta, la validez conceptual y los problemas político-epistemológicos que surgen de algunas formas de captación y análisis de lo que, desde nuestra perspectiva teórica, podemos denominar capital cultural (Bourdieu 2011; Gutiérrez 2012) en el marco de su puesta en juego en prácticas laborales de jóvenes de sectores populares. Esta discusión conceptual presupone que la construcción teórica de problemáticas dispone y produce relaciones y objetos de intervención realmente construidos, con lo cual la preocupación epistemológica por los alcances y consecuencias del uso de categorías - como la de capital cultural- es, a su vez, una preocupación eminentemente política y práctica.

Para esto, el trabajo comenzará presentando la lógica conceptual de la categoría de «capital cultural» en el marco de la teoría de las prácticas de Pierre Bourdieu. Luego, se analizará el lugar específico del capital escolar en esta construcción teórica, para revisar algunos datos estructurales de la distribución de este recurso de poder y de la dinámica del mercado laboral juvenil en general. Posteriormente, pondremos en juego estas categorías para analizar el caso de un grupo de jóvenes de un barrio popular de la Ciudad de Córdoba, recuperando la perspectiva crítica de los estudios de cultura popular - fundamentalmente los aportes de Grignon y Passeron (1998) - en orden a reinsertar el potencial interpretativo del reconocimiento de las discontinuidades «verticales» y «horizontales» de las lógicas de inversión de capitales en el espacio social. Para finalizar, plantearemos algunas de las posibilidades abiertas por esta revisión conceptual para la eficacia y profundidad de la intervención de políticas de empleo para jóvenes en nuestro país.

en distintos espacios públicos e instituciones del barrio. Los nombres y datos de lugares fueron modificados u omitidos para guardar la identidad e intimidad de las personas. Aquí particularmente trabajaremos con las trayectorias de los jóvenes. El trabajo tiene lugar en el marco de una investigación para mi tesis doctoral, sobre la cultura del trabajo en jóvenes de sectores populares en el contexto de la postconvertibilidad argentina. 


\section{LA CULTURA COMO RECURSO (PARA EL TRABAJO)}

Con una lente preformada por el diagnóstico político sobre la cuestión (Weller, 2008), ${ }^{3}$ nuestra forma de pensar las diferencias entre las distintas estrategias laborales llevadas a cabo por los jóvenes estaba fuertemente influida por el supuesto de que el capital cultural era uno de los recursos fundamentales para desplegar estrategias laborales «exitosas», en términos de estabilidad, de posibilidad de capitalización económica, de reinversión, etétera. En términos generales, este postulado es difícilmente discutible. Pero al poner en juego esta categoría en las trayectorias de empleo recorridas por los jóvenes del barrio, nos encontramos con algunos problemas.

\section{ESPACIO SOCIAL Y CAPITALES}

Nuestro punto de partida teórico es la noción de espacio social. Tal como lo entiende Pierre Bourdieu, se trata de un espacio multidimensional, del cual podemos distinguir tres vectores fundamentales de construcción: el volumen de capital, la estructura del mismo y la trayectoria social tanto de la formación distributiva del capital como de los agentes (Gutiérrez, 2005; Bourdieu, 2011b). El primero (volumen) define, justamente, las diferencias fundamentales del espacio social basándose en la desigualdad de recursos entre las clases más privilegiadas y las más desposeídas en cuanto a capital económico y cultural. Mientras que éstos funcionan como los «principios de estructuración» del espacio social, el capital social y el capital simbólico funcionan como los «principios de rentabilidad adicional» de estos capitales. ${ }^{4}$

3 Es menester precisar que el caso argentino puede ser excepcional al respecto de la proporción de la población con titulación de nivel medio en el marco latinoamericano. Al respecto, sostenemos la posición de profundizar el análisis contextual y relacional de las categorías.

4 De aquí en adelante utilizaremos estos términos en el sentido que les atribuye Alicia Gutiérrez: el capital económico es, probablemente, el que Bourdieu menos se esfuerza en definir, aunque refiere claramente a la propiedad-acumulación de bienes materiales, monetarios, ingresos, etcétera. Constituye el capital dominante, así como también, en cuanto campo, tiende a imprimir su estructura en el resto de los campos (conservando éstos siempre la relativa autonomía de la que habláramos). El capital cultural será luego definido en detalle. Por su parte, «El capital social está ligado a un círculo de relaciones estables» (Gutiérrez, 2012), 
El segundo, (estructura patrimonial) en cambio, define las fracciones de clase en base a diferencias «secundarias». Esto implica que las «formas diferentes de distribución del capital global entre las distintas especies de capital» definen principios de elección, capacidades de reproducción, gustos y representaciones, claramente diferenciados entre, por ejemplo, los «productores artísticos» (poseedores de una proporción mayor de capital cultural) y los «patronos industriales» (poseedores de proporción mayor de capital económico). Dicha estructura lleva muchas veces a la producción de prácticas simbólicas diametralmente opuestas y prácticas de distinción que definen conflictivamente una y otra fracción, aunque ambas pertenezcan al «campo de poder» o «clase dominante» (Bourdieu, 2006).

La tercera dimensión, la del tiempo o la trayectoria, se vincula a la recomposición de unidades de análisis más homogéneas en términos de condiciones de producción de habitus - esquemas y disposiciones - (Bourdieu, 2010). De esta manera, la construcción del espacio social en Bourdieu implica la reconstrucción del sistema de posiciones sociales, las relaciones de fuerza trabadas entre ellas y las relaciones de sentido tejidas en torno a las posiciones - la manera en la que los agentes comprenden este espacio social y construyen conocimiento en su contexto-y sus relaciones.

\section{DISCUSIONES SOBRE EL CAPITAL CULTURAL Y LA ESCUELA}

Tal como sostienen Lamont y Lareau (1988), el concepto de capital cultural fue originalmente acuñado por Bourdieu y Passeron (1998) para analizar cómo la cultura y la educación contribuían a la reproducción social (del espacio de las clases, tal como vimos anteriormente). Tomando el criterio más clásico para pensar el capital cultural «insti-

también denominadas redes, más o menos institucionalizadas, de reconocimiento de la honorabilidad y la respetabilidad del agente y que a su vez puede generar beneficios tanto materiales como simbólicos, producto de estrategias de inversión y reproducción social. Por último, el capital simbólico alude a la acumulación de bienes como el «honor», el «prestigio»y el «reconocimiento» (posesión legítima de un capital en un campo), y es el que añade la fuerza simbólica o eficiencia mágica al uso y estrategias basadas en los otros capitales. De esta idea se deriva la interpretación de aquellas prácticas de «violencia eufemizada» que el autor nomina como violencia simbólica. 
tucionalizado», esto es, la titulación escolar, ${ }^{5}$ el campo de los jóvenes entrevistados se nos presentaba extraordinaria, aunque previsiblemente homogéneo. Como planteamos, casi ninguno había finalizado sus estudios. Algunos se encontraban asistiendo a la escuela en un Centro de Enseñanza Media para Adultos (CENMA) y otros estaban francamente fuera del sistema escolar. ${ }^{6}$ Otro dato llamaba la atención: aquellos pocos que habían finalizado sus estudios de nivel medio, no parecían presentar condiciones laborales sustancialmente mejores.

Esto nos llevó a repensar la noción misma de capital cultural. En su definición más general, el mismo está ligado «[...] a conocimientos, ciencia, arte, y se impone como una hipótesis indispensable para rendir cuenta de las desigualdades de las performances escolares» (Gutiérrez, 2012). En este sentido, la escuela aparece como la «principal instancia legítima de legitimación» de la arbitrariedad cultural que contribuye a la «reproducción de la estructura de la distribución del capital cultural entre las clases y, con ello, a la reproducción de las relaciones de clase existentes» (Gutiérrez, 2012). Por otra parte, esta definición no agota la categoría. Bourdieu distingue tres estados del capital cultural. El primero, incorporado; es decir, capital cultural hecho cuerpo, vuelto habitus. El segundo, el estado objetivado; es decir, cultura en bienes y soportes materiales, tales como cuadros, pinturas, libros, etcétera. Y por último, el estado institucionalizado, como competencia reconocida por una institución legítimamente autorizada a tal fin (Bourdieu, 2011a). Ya no en el nivel conceptual formulado por el mismo Bourdieu, sino en la aplicación empírica y práctica de esta categoría, muchas veces se prioriza la manifestación institucionalizada de este capital. ${ }^{7}$ Tal como sostienen Lamont y Lareau, el capital cultural puede ser pensado como «marcas de cultura» de alto

5 Es fundamental recordar que Bourdieu (2011b: 45) opera identificando los recursos propios del campo de poder como aquellos legítimos y válidos para el análisis de todo el espacio social de las clases. Como luego observaremos, este modo de operar presenta algunos problemas epistemológicos.

6 No podemos ahondar demasiado aquí, pero debemos mencionar al menos las particularidades de esta escolarización: en general son escuelas definidas como para «jóvenes y adultos», en modalidades avanzadas (el nivel medio en tres años), con horarios usualmente nocturnos.

7 Descontamos el hecho de que, para estudios cuantitativos, esta forma de medición presenta facilidades y posibilidades de medición claras y casi indiscutibles. 
estatus, institucionalizadas y compartidas, que son usadas para la exclusión social y cultural respecto de trabajos, recursos y grupos de estatus (Lamont y Lareau, 1988). Pero a partir de esta definición, ¿qué podemos decir del uso de la cultura entre aquellos que han quedado, efectivamente, relegados, desposeídos o incluso excluidos?

El título secundario se percibe generalizadamente como indispensable para la consecución de empleos de calidad. Este es un imaginario fuertemente arraigado en la historia nacional argentina, sobre todo a partir de la emergencia de políticas culturales orientadas a los sectores populares durante los años cuarenta y de una presencia realmente relevante de la educación pública y obligatoria en todo el territorio del país desde la consolidación misma del aparato estatal en la segunda mitad del siglo XIX (Svampa, 2005). Este ideario arraiga incluso hoy en las concepciones de los funcionarios y técnicos de las políticas de empleo juvenil, pero también en los mismos jóvenes, que rescatan esta imagen al momento de ser interrogados acerca de la finalidad con la que asisten al «acelerado». ${ }^{8}$ «Poder trabajar»y «llegar a ser alguien» como formas de dignificación personal son razones fundamentales esgrimidas fuertemente por los jóvenes con los que nos encontramos.

\section{JóVENES, CAPITAL CULTURAL Y TRABAJO}

\section{a) El estado del mercado laboral para jóvenes}

Entre el año 2003 y el año 2010, la mayoría de los indicadores laborales tendieron a mejorar, exceptuando algunos ciclos negativos a raíz de las crisis internacionales (como los del bienio 2008-2009). La mayoría de los análisis coinciden en que, a grandes rasgos, el desempleo y subempleo disminuyeron de manera considerable, estabilizándose en porcentajes de una sola cifra, se restablecieron las negociaciones colectivas legalmente, reconstituyendo el poder de la trama sindical, como así también se avanzó en materia de registro del empleo (Neffa, Oliveri y Persia, 2011), aunque los números varíen de acuerdo a cómo se mida la precariedad/informalidad de los puestos (Narodowski, Panigo y Dvoskin, 2011). Muchos reconocen también un viraje de las políticas de empleo a nivel nacional, hacia el paradigma de la «activación», orientado ya no a la población desocupada, sino a la formación y orientación de la población «inempleable» (Pérez, 2013; Jacinto y Millenaar, 2009).

8 Referencia a la Escuela para adultos en el lenguaje coloquial. 
Los datos que provee el MTESS plantean una fuerte vinculación entre «trabajo no registrado»y «nivel de instrucción» ${ }^{9}$. El porcentaje de empleo no registrado asciende de 12,6\% para el nivel terciario o universitario completo y a 51,6 para los que poseen solo primario completo. Sin embargo, las particularidades de esta dinámica para los jóvenes, y específicamente para aquellos de sectores populares, ${ }^{10}$ debe ser analizada a escala para comprender algunos de los problemas que surgen de la complejidad del análisis empírico. Como franja de edad, los jóvenes entre 15 y 24 años o entre 18 y 25 -de acuerdo al corte aplicado por las estadísticas oficiales y las políticas de empleo- son uno de los grupos que de manera más importante están afectados por los fenómenos de la informalidad, la precariedad, la inestabilidad y el desempleo. Y, si bien en términos absolutos también para esta franja las cifras mejoraron, la brecha entre «jóvenes» y «adultos» creció paradójicamente durante el período. Mientras que, según datos de CEDLAS, para el año 2003 el desempleo de la población entre 15 y 24 años superaba en 2,8 veces al de la población entre 25 y 64 años, esa cifra asciende a 3,6 veces en el año 2010. Según la misma fuente, para el año 2010, los asalariados sin derecho a jubilación del primer grupo ascienden a un 56\%, mientras que constituyen un $30 \%$ del segundo grupo de edad. Una relación similar de desigualdad podemos establecer en términos de asalariados sin cobertura de salud por la ocupación principal.

En el conglomerado de Gran Córdoba, de acuerdo a los datos que provee la Encuesta Permanente de Hogares (EPH) para el tercer trimestre de $2011,{ }^{11}$ encontramos que los valores modales para el «carácter

9 Si bien consideramos que esta relación tiene una significación importante, entendemos que solo puede visualizarse si abandonamos un modelo de causalidad bivariada e intentamos captar la complejidad de la causalidad estructural en la conformación de las prácticas sociales enclasadas; es decir, la multidimensionalidad del espacio de posiciones que las condiciona y define como campos posibles. Para un desarrollo de este argumento, ver Baranger (2004).

10 Para subgrupos en general, de acuerdo a lo que plantea la literatura especializada: mujeres, trabajadores de bajos salarios, de bajo nivel de instrucción y baja calificación (Narodowski, Panigo y Dvoskin, 2011).

11 Los datos que presentaremos a continuación surgen del análisis que se realiza en el Proyecto «Las clases y su reproducción en el espacio social cordobés (2003-2013)», radicado en SECYT-FFYH-UNC y dirigido por Alicia Gutiérrez y Héctor Mansilla. Concretamente utilizaremos los da- 
ocupacional» de los jóvenes se ubican en el comercio (9\%) y en la gestión administrativa y jurídica $(5,91 \%)$. Sin embargo, si consideramos específicamente a los jóvenes provenientes de hogares de sectores populares estos valores aparecen en comercio (13\%) y construcción $(11 \%),{ }^{12}$ mientras que los de sectores dominantes se ubican mayormente en puestos directivos (18\%).

Por su parte, mientras que los valores para «desocupación»e «inactividad» no tienen variaciones tan fuertes asociadas a los sectores de los hogares de los que los jóvenes provienen (varían, respectivamente, de $6,8 \%$ a $5,4 \%$, y de $43 \%$ a $54 \%$, de sectores populares a sectores dominantes), sí se establecen grandes diferencias en las categorías de inactividad en las cuales los jóvenes se distribuyen: mientras que los valores para la categoría «estudiante» son de $94 \%$ y $85 \%$ para los sectores medios-altos y dominantes, disminuyen a $58 \%$ o $47 \%$ en los sectores populares y medios-bajos. Lo mismo, aunque en sentido inverso, sucede con la categoría «amas de casa» con $5 \%$ para sectores dominantes y $26 \%$ para sectores populares.

Por último, podemos señalar que, mientras que para los jóvenes en general un $65 \%$ completó sus estudios secundarios (o superiores),

tos de un Análisis de Correspondencias Múltiples a partir del cual se construye el Espacio Social de las Clases para Gran Córdoba con las estadísticas disponibles en las bases individuales y de hogares del INDEC. Aunque no contamos con espacio aquí para realizar una descripción detallada de esta técnica (apropiada para el pensamiento teórico, epistemológico y metodológico relacional), debemos mencionar que su objetivo es justamente contribuir a identificar algunas asociaciones básicas en la configuración del sistema de posiciones y relaciones entre posiciones a partir de los múltiples recursos fundamentales y su distribución, los cuales constituyen la estructura de las clases sociales. Para este trabajo los datos funcionarán sólo a modo de graficar la distribución de los recursos y representar un estado de relaciones de fuerza, sin volverse el centro de nuestro argumento. Hablaremos, a partir de este análisis (provisorio y en curso) de cuatro clases fundementales en términos de sectores de este espacio social: sectores populares, sectores medios-bajos, sectores medios-altos y sectores dominantes. Para una descripción más detallada de la técnica y del espacio social construido Ver Gutiérrez y Mansilla (2013). Los principios teórico-metodológicos de la técnica pueden encontrarse en Baranger (2004).

12 Habría que agregar, en este caso, a Servicio Doméstico, que asciende a $4,54 \%$ y $5,54 \%$ para sectores populares y medios-bajos, concentrando gran parte de las mujeres ocupadas en estos grupos. 
lo hizo un $73 \%$ de los jóvenes provenientes de hogares de sectores dominantes y un $42 \%$ de los jóvenes provenientes de sectores populares. El porcentaje de los jóvenes que poseen como máximo nivel de instrucción a la educación primaria completa o inferior es del $9,77 \%$ en general, mientras que es de $2,7 \%$ y $26,6 \%$ respectivamente para los jóvenes provenientes de sectores dominantes y populares.

En términos generales, a partir de estos datos, podemos visualizar una distribución desigual de los recursos estratégicos en el espacio social de las clases de Córdoba, y al mismo tiempo, observamos cómo las estrategias de jóvenes de sectores medios-altos y dominantes definen una acumulación en términos de capital escolar, que estructura además una inserción en los puestos mejor posicionados en el mercado laboral: protegidos, cualificados, con aportes, obra social, etcétera. Volviendo a lo adelantado acerca de la determinación de la inserción por el nivel de instrucción, podemos observar que lo que se pone en juego es más bien el peso estructural de una diversidad de determinaciones que configura un espacio social multidimensional. Si con este mapeo podemos definir los grandes principios estructurantes de las desigualdades en el espacio, no debemos olvidar que las composiciones patrimoniales y las trayectorias modales de clase establecen criterios más específicos de disputa y distinción en el marco de los distintos sectores de este espacio. Esto nos lleva a pensar las disputas, diferencias y distinciones hacia el interior del espacio de los jóvenes de sectores populares, y la reconfiguración de recurso cultural en este marco.

\section{BREVE DESCRIPCIÓN DE LOS ENTREVISTADOS}

Los trayectos de los jóvenes presentan muchas de estas recurrencias. En primera instancia, podemos aclarar que utilizamos la noción de «popular» como un recurso teórico que permite construir una lógica general de gestación cultural coherentemente con un conjunto de datos empíricos (desempleo o empleo precario, ocupaciones no calificadas, desposesión de capital cultural «legítimo», ingresos inestables y bajos, des-protección social, etcétera): «[...] las culturas populares serían los sistemas de representación y prácticas que construyen en interacciones situadas quienes tienen menores niveles de participación en la distribución de los recursos de valor instrumental, el poder y el prestigio social, y que habilitan mecanismos de adaptación y respuesta a estas circunstancias, tanto en el plano colectivo como individual» (Míguez y Semán, 2006). Al mismo tiempo estamos nombrando, con 
esta categoría, un contenido más concreto, histórico y circunscripto, referente a una tradición, unos recursos, unos contenidos y unas transformaciones particulares de la vida de las clases populares en nuestro país: valores culturales muy arraigados en torno a las instituciones de la Escuela, la Familia y el Trabajo.

En el caso de los varones, todos han trabajado en la rama de la construcción. Algunos contribuyendo para la edificación de su propia vivienda, la gran mayoría participando en esta actividad como primer trabajo, a los 14, 15 ó 16 años, período que coincide con momento en el que muchos «dejaron» la escuela secundaria. Salvo casos muy puntuales, en los que los jóvenes se presentaron en obras de la zona céntrica de la ciudad a ofrecer su fuerza de trabajo como «desconocidos», el resto de las veces consiguieron el puesto por contactos de sus padres o fueron empleados por sus vecinos o sus tíos. Esto marca también una fuerte continuidad en las trayectorias respecto de las ocupaciones de sus progenitores, a pesar de que los jóvenes han tenido siempre un recorrido escolar más prolongado que el de sus padres: la gran mayoría, con algunos grados diferenciales de calificación, son o fueron también albañiles. ${ }^{13}$

En el caso de las mujeres, también es recurrente un tipo particular de ocupación: el trabajo doméstico. Ya sea cuidando niños o realizando tareas de limpieza, todas han participado de este tipo de actividades, y de igual manera que los varones, han accedido a estos puestos a partir de contactos familiares, de vecinos, amigos, o de la iglesia o templo. Con menor frecuencia, han participado también en tareas de atención al público en pequeños comercios.

13 Esta tendencia puede encontrarse también en el estudio de Alicia Gutiérrez, aunque referida al contexto de los noventa: «Hay, claramente, mayor inversión en capital escolar de los hijos, al menos de algunos de ellos, que muestran una trayectoria en la escuela más amplia que la de sus padres (y que es similar a la que tienen las familias de la segunda generación). Sin embargo, no existen diferencias entre ambos en el tipo de ocupación, sino que, más bien, los hijos parecen estar destinados socialmente a seguir las distintas etapas que caracterizan la trayectoria laboral de los padres. Es más, en el marco del aumento de las tasas de desempleo, la oferta de puestos de trabajo incrementa los requisitos educacionales para cubrir vacantes» (2005). Luego intentaremos dar cuenta de este supuesto desarreglo entre el aumento del capital escolar y la continuidad en cuanto a las categorías ocupacionales. 
Ninguno de los jóvenes entrevistados ocupa el rol de Jefe de Hogar. Tampoco poseen un empleo registrado, por lo que no cuentan con obra social, y prácticamente ninguno cuenta con cobertura por parte de sus padres (quienes se encuentran en la misma situación). Casi todos viven en la casa de su familia de origen, salvo dos mujeres, con hijos, que actualmente conviven con sus parejas. Todos terminaron la escuela primaria, y solo dos de ellos terminaron la secundaria.

\section{LAS HISTORIAS LABORALES Y LA DISTRIBUCIÓN DE CAPITAL CULTURAL ENTRE LOS JÓVENES}

Ambos padres de Alejandro trabajan en el campo de la salud como enfermeros, y poseen un título terciario que los habilita para dicho ejercicio. La casa en la que viven fue adquirida a través de una cooperativa de vivienda $\mathrm{y}$, con el tiempo, la fueron ampliando: «Cuando llegamos, era una tapera», cuentan. Cuando terminaron la secundaria, migraron a la Ciudad de Córdoba desde el interior de la provincia de Entre Ríos, para realizar sus estudios. Si bien no tiene su título de la escuela media, Alejandro terminó de cursar sus estudios. Por haberse cambiado de especialidad (de electrónica a mecánica) en la escuela técnica a la que asistía, le quedaron equivalencias por rendir. Además de realizar «entrenamientos laborales» ${ }^{14}$ en el marco del Programa de empleo del que forma parte, brinda servicios de sonido para fiestas de quince y casamientos. Gran parte de los equipos los armó él mismo, a partir de sus conocimientos en electrónica. Su hermana es, según términos utilizados por sus padres, una «bocha» ${ }^{15}$ y quiere estudiar ingeniería en sistemas en la Universidad Tecnológica Nacional: «Dicen que ahí son mucho más contemplativos con los que trabajan».

14 La figura de «entrenamientos» en el marco de las políticas de empleo implica una forma de práctica laboral no regida por la normativa de la «relación de dependencia», pero además orientada en torno a un fin formativo y de capacitación. Esto, a su vez, involucra un compromiso asumido por parte del beneficiario, la empresa y el Estado, de construir un ámbito propicio para que esto pueda ser posible. Sin embargo, como pude observar en las entrevistas a distintos actores, estos cortes tan abruptos entre lo que implica la realidad de un «trabajo» y la de un «entrenamiento» son mucho más factibles en la letra del programa que en su efectiva aplicación.

15 Forma coloquial de nombrar los «buenos alumnos»y los «inteligentes». 
Leandro «dejó» la secundaria en segundo año. Tiene 23 años. Su hermana más grande tiene 31 años y tres hijos. «Terminó la escuela de grande», en la «nocturna» ${ }^{16}$ y ahora «estudia enfermería en la villa», que es un instituto de nivel terciario que funciona en una escuela pública del barrio. Su hermana más chica tiene dos hijos (es menor que Leandro) y no terminó la escuela. Su madre terminó solamente la escuela primaria. Sobre su padre no hay mayores datos, pues abandonó el hogar cuando él era todavía muy pequeño. La madre de Leandro trabajó de empleada doméstica y realizó durante un tiempo contraprestaciones laborales en una escuela por el Plan Jefes y Jefas de Hogar Desocupados, ${ }^{17}$ pero en el último tiempo enfermó de su espalda y no pudo trabajar más. Ella y Leandro viven con la segunda hermana, de 28 años, en una piecita que lograron construir al fondo del terreno que ocuparon. Él ha trabajado como albañil, pero prefiere no seguir haciéndolo: «Su cuerpo no da para un trabajo tan pesado», me dice su madre, haciendo referencia a su contextura menuda. Empezó trabajando en la empresa de limpieza de autobuses en la que actualmente se desempeña su hermana, pero luego de una semana lo despidieron, junto a todos los recién contratados, por «exceso de personal». No le resulta sencillo conseguir trabajo, pero no siente que no haber terminado la secundaria lo limite en la búsqueda.

Quiere trabajar en obras de countries (condominios) como su cuñado, pero para esto necesita un certificado de buena conducta, y como posee una contravención por «merodeo» en el centro de la ciudad, no puede acceder al mismo. ${ }^{18}$

16 Forma coloquial que refiere a la escuela para adultos.

17 En adelante, PJJHD. Este plan forma parte de las políticas sociales de la era neoliberal que, durante la crisis de 2001-2002 se masificó, hasta llegar a más de un millón y medio de beneficiarios. Para un estudio más detallado, ver Andrenacci, Ikei, Mecle y Corvalán (2006).

18 En la Provincia de Córdoba, el Código de Faltas, legislación que rige las contravenciones, habilita prácticas de detención y represión permanentes de jóvenes pobres a partir de figuras arbitrarias como la del «merodeo», que dejan a criterio de la policía cuando la permanencia o circulación de un ciudadano por determinados espacios puede ser tildada de «peligrosa» o «sospechosa». Luego, los «certificados de buena conducta» otorgados por la misma institución policial, pueden ser negados a aquellos que posean contravenciones por ese tipo de figuras contravencionales (no penales, es decir, no judicializadas). 
Luis tiene 23 años. Vive con sus padres. Su padre es pintor de obra y su madre «trabaja en la casa», manera en la que se refiere a que se emplea en servicio doméstico. Su hermano es policía y le consiguió un puesto de guardia de seguridad en el que duró cerca de dos meses. No quedó efectivo, sin entender por qué. Antes trabajó en fábricas y empresas comerciales medianas. También trabajó en la recepción de un centro de salud privado del barrio. Según su propia evaluación, le costaba mucho atender al público. Es uno de los únicos entrevistados que finalizó el nivel medio de educación en la escuela parroquial del barrio. A pesar de contar con el recurso de la titulación, que en ninguno de los otros casos de varones aparece, no logra sostener un trabajo en el tiempo, y esto lo frustra mucho.

Mirta tiene 25 años. Vive frente a la casa de su madre, con su marido (trabajador de una fábrica automotriz) y su hijo de 4 años. No forma parte del mismo programa que el resto. Desde hace varios años es profesora de danzas, disciplina que estudia desde que tiene nueve años. Su madre es la pastora de una iglesia evangelista en un barrio cercano, y durante mucho tiempo sostuvo copas de leche y hogares para niños. Además, Mirta también trabaja como niñera, cuidando a su sobrino. Hace unos años realizó un curso de estilista, por lo que cada tanto realiza tareas de depilación y estética corporal a pedido. Su única experiencia de empleo registrado en relación de dependencia fue un trabajo temporal en un bar de un centro comercial, en el que trabajó una temporada cubriendo una licencia. Después tuvo su hijo y con su propia academia de danza logra organizar los horarios y articular para poder cumplir con su función de «madre» $\mathrm{y}$ «esposa».

Inés tiene 22 años y un hijo de 4 . Vive con su abuela, luego de varios desplazamientos entre la casa de su madre, la de su padre y la de otra abuela, en el mismo terreno que su padre y su tío, cada uno con sus respectivos grupos familiares nucleares. Empezó varias veces la escuela para adultos, pero la abandonó. Dice que ahora solo quiere anotarse cuando esté segura de que lo terminará. Comenzó a trabajar a los doce años, en la casa de una «conocida del salón» (un templo de Testigos de Jehová). También cuidó, durante un tiempo, los hijos de otro miembro del salón. Siempre trabajos cortos e interrumpidos. Estuvo trabajando dos meses en una pizzería, de encargada de la caja, pero en la reducción de personal quedó fuera. Pretendía ingresar a trabajar a un Call Center, pero siempre quedaba fuera en los procesos de selección de personal. Actualmente no está buscando trabajo. 
Alejandro puso en juego una estrategia de acumulación de capital global (cultural y económico) colectivamente articulada junto a su familia. La posibilidad de terminar la escuela media implicó un compromiso de su parte, pero también de parte de sus padres, de dispensarlo de tener que ingresar de lleno al mundo laboral a edad temprana. ${ }^{19}$ Por otra parte, el dinero que obtuvo a partir de sus trabajos temporales fue guardándolo (entregándoselo a su madre, para no gastarlo) e invirtiéndolo. Primero compró los elementos y armó los equipos de sonido. Luego, con el dinero obtenido en los servicios de fiesta, le compró un Ford Sierra a su padre, a partir de lo cual la familia se vio posibilitada para cambiar el auto por un modelo cero kilómetro.

Al igual que Alejandro, a Leandro le interesa la mecánica del automotor. Dice que no le molesta realizar cursos, pero prefiere otra vía: «Me doy maña». Alejandro utiliza la misma expresión. Este último está actualmente trabajando, luego de un largo «entrenamiento», ${ }^{20}$ en un taller de chapa y pintura. Además, se recibió de la escuela técnica con la especialidad en mecánica. Leandro, como Luis, en cambio, se encuentran actualmente desempleados.

Inés siente cierta limitación en sus posibilidades laborales por su carencia de título secundario, pero siente más frustración por empezar «el acelerado» y dejarlo a la mitad. La actual posición de Mirta, en cambio, se debe en gran medida a sus competencias puestas en juego en el ámbito laboral: un saber acumulado e incorporado desde los 9 años, que desempeña en diversos ámbitos. También cuenta con la certificación de finalización de sus estudios secundarios, más allá de que no la pone en juego para lo que considera sus «logros» en el ámbito laboral y económico.

19 Gutiérrez analiza de esta manera la cuestión de la «obligación» de asistir a la escuela primaria: «Esta «obligación» implica una serie de prácticas y de representaciones asociadas: el compromiso de los padres de hacerlo posible, especialmente retardando el ingreso de los hijos al mercado laboral (comienzan a trabajar a los 13 ó 14 años, cuando terminan el útlimo grado del nivel primario de educación formal), y con ello, de hacerse cargo de todos los gastos que se ocasionan, y el de los hijos, de reconocer y valorar el esfuerzo paterno cumpliendo con las tareas escolares, en lo posible año tras año, «grado tras grado», «sin repetir ninguno» (Gutiérrez 2003:124).

20 Prestación similar a una pasantía en el marco del PJMYMT. 


\section{LA (DIS)CONTINUIDAD DEL ESPACIO SOCIAL Y DE LAS HERRAMIENTAS CONCEPTUALES}

\section{a) Algunos supuestos teóricos para los estudios de culturas populares}

Un abordaje como el que pretendemos, orientado por la perspectiva de los estudios de culturas populares, que permita desentrañar los problemas empíricos que se nos presentan en el uso práctico de la categoría de capital cultura, implica un esfuerzo por reconstruir de manera compleja la mirada sociológica que articula el principio de dominación; es decir, la «homogeneidad vertical» para el pensamiento de las relaciones en el espacio social de las clases, con el principio de autonomía, o bien, el reconocimiento de las discontinuidades, segmentaciones y heterogeneidades al interior de las clases dominantes y dominadas (Grignon y Passeron, 1992).

Por otra parte, esta perspectiva implica, para la construcción de un análisis crítico, una serie de recaudos teórico-metodológicos. En primer lugar, la necesidad de un análisis que dé cuenta al mismo tiempo de la dimensión ideológica (es decir, la especificidad de los elementos simbólicos en la relación de «dominación social» entre clases) y de la dimensión cultural (es decir, el sentido de las prácticas no solo en relación a sus condiciones, sino también en relación a la constitución de simbolismos en términos de «cultura de clase» y a los puntos de contacto, negociación, disputa e impugnación de otras culturas de clase). Esto significa reconocer que «[...] le pasan cosas esenciales a una cultura popular como consecuencia de estar restringida a funcionar como cultura dominada, es decir, inextricablemente como cultura de denegación y como cultura de aceptación, sub-cultura y contracultura. La sociología debe ocuparse de eso: es análisis ideológico... Pero el sociólogo olvidaría algo de la cultura popular si no fuera capaz de hacer la hipótesis interpretativa de que una cultura popular es también capaz de productividad simbólica cuando olvida la dominación de los «otros», a menos que llegue a organizar, de manera simbólicamente coherente con su principio propio, a las experiencias de su condición: es análisis cultural [...] (Grignon y Passeron, 1992).

Es así que, a partir de nuestro análisis de las prácticas laborales debimos dar cuenta de una serie de saberes, competencias y haberes en los jóvenes que, medidos con la regla de las «competencias legítimas», eran homologados, abstraídos o pasados por alto, como realiza- 
ción de una forma de pensamiento sociocéntrica y de una violencia epistemológica contra las clases populares: la de ver allí, entre los dominados, solo la carencia, o la falta de aquello que buscamos con la lente construida para medir y reconocer la complejidad y la singularidad de lo dominante. Dispuestos con las armas de la «teoría legitimista de la cultura» nos encontramos en cierta forma limitados para la descripción y la explicación de algunas de estas diferencias. Sin embargo, esto no es responsabilidad exclusiva de la formulación teórica. De hecho, la noción de capital exige siempre un esfuerzo por su contextualización y por su aplicación situada y relacional. Pero por otra parte, en el intento de reconocer el proceso de dominación que implica la monopolización del carácter legítimo de la cultura en manos de las clases dominantes, la sociología puede caer en una definición de las culturas populares como «pura carencia», en un análisis que presenta como herramienta de medición un entramado conceptual diseñado para la descripción densa y profunda de las diferencias al interior del campo de poder. ${ }^{21}$ Pero, ¿Qué sucede cuando la sociología intenta dar cuenta de las complejidades de las experiencias y prácticas simbólicas de las clases populares?

El proceso de homogeneización «vertical» que se produce con una categoría como la de capital cultural para reconocer el vector de la «estratificación social», produce fundamentalmente entre los sectores populares una homogeneización «horizontal», es decir, un desconocimiento de las diferencias tramadas al interior de estos sectores, de su constitutiva fragmentación y heterogeneidad. A partir de esto, Grignon y Passeron (1992) reconocen que esta discontinuidad debe corresponderse con una escisión conceptual como uno de los momentos de la investigación en pos de analizar diferencias significativas entre fracciones de las clases populares. En este sentido, Grignon sostiene que puede pensarse en el «pequeño patrimonio» como una forma de capi-

21 De hecho, tal como plantean Lamont y Lareau, en algunas de sus formulaciones, la noción de capital cultural aparece prácticamente como equivalente de «cultura legítima»; es decir, ya sea como cultura «más valorada», o bien como cultura «respetable». De cualquier forma, los autores asumen que las marcas de estatus cultural alto entre las clases bajas juegan la misma función (excluyente) que la cultura legítima realiza en las clases medias y altas (Lamont y Lareau 1988:157). Aquí quisiéramos mostrar que, si bien en un nivel formal esto puede ser cierto, los desplazamientos e instanciaciones empíricas son sumamente complejas $\mathrm{y}$ adquieren lógicas y tendencias propias de sus contextos singulares. 
tal económico, en las «funciones desempeñadas por los agentes populares en su medio» como un equivalente del capital social en el mundo popular, y en las «culturas de oficio» como la forma específicamente popular del capital cultural. «Considero solamente que la oposición entre el pequeño patrimonio, la pequeña propiedad residual y los saberes profesionales o las certificaciones escolares pueden tener el mismo papel en un estudio comparativo de los gustos de las diferentes fracciones de la clase obrera que la oposición entre las diferentes clases de capital en el estudio comparativo de los gustos de las diferentes fracciones de la clase dominante» (Grignon y Passeron, 1992).

\section{LÓGICAS DIFERENCIALES EN LA INVERSIÓN DE CAPITAL CULTURAL}

Si bien la discusión entre estos autores advierte sobre el riesgo de «miniaturizar» los haberes populares reconociéndolos como equivalentes de recursos dominantes, pensados aún a partir del formato de la categoría de «capital», ${ }^{22}$ sus señalamientos fueron sumamente útiles para (re)pensar nuestro propio material empírico y analizar críticamente algunas orientaciones contemporáneas en la política de empleo nacional.

Por un lado, habilita reconocer los efectos diferenciales de los títulos escolares en las clases populares y en las clases dominantes. Por el otro, señala la diferencia entre las diferencias: entre los «títulos de alto nivel» (de grado y postgrado) y los «títulos de bajo nivel»

22 Un ejemplo de la posibilidad de sortear de manera empírica estos problemas es la investigación de Alicia Gutiérrez (2005) en donde se intenta dar cuenta de otros recursos en términos de «capital habilidades»o «capital información». Otro caso es el del citado libro de Grignon y Passeron (1992) en donde mencionan las «culturas de oficio» para hablar de capital cultural en sectores populares. Si bien la categoría puede ser productiva en determinados ámbitos, una característica que complejiza nuestro campo es el hecho de que si bien la clase y el trabajo no han perdido su importancia como elementos explicativos de la estructuración de la vida cotidiana de los jóvenes (a contramano de lo que se intentó plantear desde algunos discursos académicos durante la década del noventa), las instituciones que rodean y unifican la experiencia de clase rara vez se encuentran en nuestro caso, o bien, carecen de la presencia necesaria como para poder clasificar con categorías que implican tal continuidad o tradición como una «cultura de oficio». 
(primarios y de nivel medio, completos o incompletos). A partir de estas ideas llegamos en nuestra investigación a un recordatorio acerca de la necesidad de pensar no solo en los capitales y en su desigual distribución, sino también en las condiciones necesarias para su valorización, en el estado de desarrollo de los instrumentos de reproducción (Gutiérrez, 2005) y, en relación con esto último, en su habilitación a partir de las propiedades legítimas incorporadas para valorizarlos, es decir, a partir del habitus.

Tal como sostienen Grignon y Passeron: «Estos capitales no comparten ni los «campos» ni los «mercados» en los que operan (mercado de trabajo, de esparcimiento, de matrimonio, de sociabilidad), ni las funciones por medio de las que operan (modeladas por representaciones diferentes de la legitimidad cultural, incluso por su olvido o su denegación), ni, evidentemente, sus ingredientes simbólicos y técnicos ni su «composición orgánica» (1992).

El peligro para la interpretación estriba, justamente, en un análisis de la estructura patrimonial (los recursos con los que cuentan los agentes para poner en juego en sus estrategias de reproducción social) desanclado de las estructuras incorporadas que funcionan como disposiciones para la práctica. Luis posee actualmente certificación de su escolaridad media finalizada. Sin embargo, sus búsquedas laborales siguen orientadas por contactos y familiares, para los cuales su titulación no es tan relevante, o bien, no parece valorizarse de manera particular. La trayectoria de Leandro presenta elementos similares, aunque sin la titulación. Su búsqueda se orienta a partir de familiares, y su limitación más importante para conseguir un puesto deseado (como albañil en las obras de barrios cerrados o condominios) es otro tipo de certificación: no la de su competencia y conocimiento, sino la de su (buena) conducta. ${ }^{23}$

23 El denominado «Certificado de buena conducta» es un comprobante expedido por las instituciones policiales garantizando la inexistencia de antecedentes penales o contravencionales por parte del solicitante. Dada la complejidad del aparato represivo y la inconstitucionalidad de los códigos que rigen las figuras contravencionales, incluso para aquellos jóvenes que no cometieron delito alguno se vuelve difícil conseguir esta certificación: muchos de los jóvenes fueron detenidos por figuras ambiguas como «merodeo» (circular sospechosamente por espacios públicos) o «escándalo en la via pública» (beber alcohol en la vereda de algún lugar). En este sentido, la certificación funciona mucho más como un mecanismo de selección social que como un mecanismo de certificación penal. 
Las primeras experiencias laborales, y en gran medida la continuidad respecto de las experiencias de sus padres, sedimentan en un sistema de disposiciones a (y para) la práctica que difícilmente se altere a partir de la consecución del título de la escuela media, y que por su histéresis tiende a realizar sus apuestas en los espacios de juego en los que históricamente se vio involucrado (el joven y su familia). ${ }^{24}$ Aquí primaría la estructura patrimonial antes que el aumento global (a partir de la certificación de estudios) de capital. Este ámbito de apuesta es fundamentalmente para los puestos de trabajo informales, ${ }^{25}$ en cuyos procesos de selección, los «contactos» y la «reputación» apare-

24 Un ejemplo claro de esta dinámica es el de la investigación de Gabriel Kessler (2000) sobre las experiencias de empobrecimiento de los sectores medios durante los años noventa, en donde el autor muestra la manera en la que las estructuras incorporadas, en su tendencia a permanecer, reproducían prácticas que ya no se relacionaban con las actuales condiciones materiales de vida de los agentes: enviar a los hijos a una escuela privada, incluso cuando los recursos fueran insuficientes para realizarlo, endeudándose o privándose de otras cuestiones vitales. Esto, por su parte, no implica excluir el análisis de los procesos de reflexividad que se disparan para las redefiniciones de las nuevas situaciones a las que se veían arrojados los agentes por los procesos globales de empobrecimiento

25 La informalidad del empleo, si bien se redujo en el país, permanece en niveles altos, constituyéndose en un problema de carácter estructural. Los métodos en su medición varían: algunos autores sostienen que el «empleo no registrado» llega al $36 \%$ de los asalariados para el año 2008, aunque, teniendo en cuenta la recuperación en la participación de los «cuentapropistas» en proporción a la PEA, y que por otra parte el crecimiento de esta condición de actividad no es acompañado por una recuperación en sus niveles de ingreso, el denominado Sector Informal Urbano (de acuerdo a la perspectiva de la Organización Internacional del Trabajo) llega al 55\% de la misma (Neffa, Oliveri y Persia, 2011). Además, la tasa de empleo informal afecta en mayor medida a subgrupos, entre los cuales podemos encontrar tanto a «jóvenes», como a sectores de «bajo nivel educativo» y de «bajos salarios» (Narodowski, Panigo y Dvoskin 2011). Otros investigadores, intentando dar cuenta de la complejidad de la situación de informalidad, plantean que el aumento de demanda laboral durante este período (2003 - 2012) está basado en una mayor utilización de la capacidad instalada, con lo cual muchas veces cambiaron los pesos relativos de las distintas condiciones de informalidad: creció el «trabajo precario», en detrimento del «trabajo indigente» y de los «planes sociales» (Salvia, Fragulia y Metlika, 2006). 
cen como recursos mucho más valiosos que las competencias institucionalmente reconocidas. ${ }^{26}$ «Las referencias que pueden dar los parientes, amigos o conocidos, suerte de certificaciones de buena conducta y de habilidades incorporadas para hacer cierto tipo de tareas, y, en un sentido, capital social objetivado, circulan entre empleados y patrones como un capital necesario y suplementario del capital incorporado de quien está buscando un trabajo» (Gutiérrez, 2005).

El caso de Alejandro puede leerse desde una lógica similar. Su familia cuenta con un fuerte patrimonio cultural institucionalizado (madre y padre con títulos terciarios), puesto en juego también en sus ocupaciones (en el ámbito de la salud). Es uno de los pocos casos en que, a los veinte años, no ha superado el máximo nivel escolar alcanzado por sus padres. Sin embargo, su apuesta laboral se centra en su calificación por su asistencia a una escuela «técnica», su conocimiento en «oficios» (electrónica y mecánica) y su disposición para el trabajo «rudo». Además, a partir de sus ingresos, su acumulación incluye cierto capital tecnológico y económico que pone en juego también en sus estrategias laborales (equipos de sonido y un auto propio para trasladarlo).

Mirta cuenta con su título secundario, pero sus estrategias laborales están focalizadas en el campo de la enseñanza de la danza. La reputación de su madre le resulta fundamental, más allá de que ella utilice su parentesco adrede o no, para la construcción de la confianza social necesaria para trabajar con niñas pequeñas que quedan a su cargo. Su competencia, incorporada, aunque no reconocida por ninguna institución legitimada por el poder simbólico estatal, le permite una práctica (o más bien, varias prácticas: academia, cuidado de niños, clase de ritmos en gimnasios, servicios de estética, etcétera) que ella misma gestiona para articular con sus funciones domésticas. Sus limitaciones estriban, justamente, en la inestabilidad de sus ingresos y en su monto. Sin embargo, la ocupación de su marido le otorga cierto margen como para que esta apuesta sea una opción, en última instancia, capitalizable (aunque le impone, obviamente, limitaciones al interior de las relaciones de fuerza en el ámbito doméstico).

Inés, en cambio, es una de las pocas entrevistadas que considera que encuentra un límite en su carencia de titulación: en las selecciones

26 Un proceso similar podemos encontrar en el estudio de Patricia Vargas (2005) sobre las relaciones en el marco del trabajo «en la obra» de los obreros de la construcción. 
de personal, los call center en los que aspira a trabajar le exigen que haya terminado la escuela secundaria. Sin embargo, habría que analizar más en detalle las competencias lingüísticas que terminan definiendo las diferencias entre quienes logran ingresar y quienes quedan en el camino, aún con títulos secundarios. Habría que pensar, además, qué de la experiencia escolar en su dimensión más disciplinaria y moral aparece puesta en juego, más allá de la certificación, en las prácticas laborales de estos jóvenes.

\section{REFLEXIONES FINALES}

a) Sobre las lógicas diferenciales de los recursos culturales populares

El desconocimiento de los recursos y las elaboraciones simbólicas singulares que los agentes de sectores populares ponen en juego en sus prácticas no implica solamente una falta a la profundidad y al carácter crítico del conocimiento sociológico, sino que conlleva problemas políticos concretos. De hecho, estas dimensiones (la de la política y la del saber), son solo distinguibles analíticamente, dado que los campos de intervención (como el de la inserción laboral juvenil) son invenciones históricas en el marco de tradiciones culturales y políticas locales apoyadas en formas de conocer y construir problemas sociales.

Quisimos, con nuestro texto, contribuir al reconocimiento de las lógicas diferenciales de los repertorios culturales que los jóvenes de sectores populares ponen en juego en el ámbito de estrategias laborales concretas. A partir de esto, debemos señalar una serie de problemas que se abren en torno a la discusión conceptual que intentamos dar.

Por un lado, el problema de la política de empleo. Como venimos planteando, las intervenciones estatales que abandonan el pensamiento relacional al momento de producir políticas pierden el potencial de transformación de la distribución de poderes sociales. En este sentido, el análisis político debe incorporar en sus diagnósticos la persistencia de las estructuras subjetivas de los agentes (habitus) más allá de la acumulación de nuevo capital cultural, y la posibilidad de intervenir a nivel estructural (en las condiciones y en las reglas de juego laborales).

Esto último introduce un segundo problema: el del mercado laboral fragmentado o segmentado. Si bien esta caracterización viene planteándose al menos desde principios de los años noventa (Murmis y Feldman, 1993), a la luz del esbozo sobre la necesidad de reconocer 
las diferencias entre las diferencias generadas por la posesión de capital cultural en el mundo popular y el campo de poder o clase dominante (sin por ello perder de vista el «principio de dominación» que constituye estos polos relacionalmente), la fragmentación se debe analizar reconociendo la multiplicidad de determinaciones que se ponen en juego en los distintos campos, y el acceso diferencial que las distintas clases sociales tienen a cada uno de ellos (y no solamente a cada uno de los capitales).

Hasta aquí, podemos observar que la intervención política sobre los volúmenes absolutos de capital cultural (servicios de certificación de estudios formales), sin tener en cuenta los accesos concretos a distintos campos laborales, las estrategias de acceso (impersonales, en un caso, y a partir de la activación de capital social familiar, en otro) y el reacomodamiento de las posiciones relativas para la reproducción de las distribuciones de los recursos sociales en disputa (el ejemplo más conocido de la devaluación de los títulos de la escuela media), tiende a la producción de prácticas de intervención estériles o poco profundas por parte del Estado.

Esto de ninguna manera implica caracterizar a la escuela como un espacio sin poder de legitimación o de capitalización para las estrategias de reproducción en los sectores populares. Sin embargo, deberían al menos repensarse los términos y las dimensiones en base a los cuales se conceptualiza este espacio, y no restringir su análisis a su mera capacidad de productora de capital cultural institucionalizado. En este sentido, mientras que algunos estudios señalaron tempranamente la importancia de la escuela para la formación cultural de la fuerza de trabajo (Willis, 1988), estudios más contemporáneos perdieron de vista la dimensión más ideológica y disciplinaria de la relación Escuela-Trabajo.

Por último, el reconocimiento de la autonomía relativa del campo simbólico popular implica la necesidad de asir conceptualmente lógicas diferenciales y singularidades que son lógicamente prácticas en términos laborales. Uno de los casos más claros y relevantes es la estrategia de «darse maña». ${ }^{27}$ En cierta forma, esta práctica condensa el resto de los problemas surgidos de la discusión conceptual.

Mientras que la lógica dominante de las estrategias laborales indica la necesidad de especialización en los distintos niveles de formación (especialización en la última etapa escolar, en el grado, postgra-

27 Forma coloquial de referirse al «escamoteo» y la resolución de problemas con los escasos recursos disponibles 
do, etcétera), la estrategia del «darse maña» reconoce una forma de respuesta ante un campo laboral que se rige por otras reglas, más inestables, menos formales, y en cuyo ámbito la diversificación y la relativa autonomía para la autogestión aparecen como estrategias eficaces (en torno a mantener o mejorar la posición social del agente). ${ }^{28} \mathrm{Sin}$ plantear que esto sea «la mejor entre las opciones», debemos reconocer que la posibilidad de generar emprendimientos combinados con trabajos fijos, servicios de sonido, conocimientos de electrónica, conocimientos de mecánica, trabajo en un taller, clases de danza, cuidado de niños, clases de ritmos en gimnasios y atención de las tareas domésticas en el propio hogar aparece, en una lógica práctica de análisis situacional más inmediatista, como una estrategia eminentemente razonable. Perder de vista la tendencia diversificante del capital cultural en las clases populares, y sus logros, implica desconocer la posibilidad de intervenir eficazmente sobre una tendencia emergente en un sector relevante de las mismas.

BuENOS AIRES (ARGENTINA), DICIEMBRE 2013

RECIBIDO: DICIEMBRE 2013 ACEPTADO: DICIEMBRE 2013

\section{REFERENCIAS BIBLIOGRÁFICAS}

ANDRENACCI, LuCIANO, Lidia IKEI, Elina MECle y AleJANDRo CORVALAN (2006): «La Argentina de pie y en paz: Acerca del plan jefes y jefas de hogar desocupados y del modelo de política social de la Argentina contemporánea». En L. ANDRENACCI (comp.): Problemas de política social en la Argentina contemporánea. Buenos Aires: Prometeo-UNGS.

28 Esto no implica, de ninguna manera, actitudes celebratorias respecto de prácticas o estilos de vida como «el rebusque», tal como podemos encontrar en algunos autores: «El rebusque puede, según esto, leerse como una contracultura de las clases populares con su orden, su estética y sus fines propios» (Mendoza, 2011). La comprensibilidad práctica de las estrategias laborales de estos jóvenes no implica una construcción autónoma con un sentido de resistencia, sino más bien lógicas relativamente autónomas de acumulación y reproducción, válidas en espacios diferenciales a los dominantes y a los construidos por las instancias públicas. 
BARANGER, DENIS (2004): Epistemología y metodología en Pierre Bourdieu. Buenos Aires: Prometeo.

BOURDIEU, PIERRE (2006): La distinción: criterios y bases sociales del gusto. Madrid: Taurus.

(2010): El sentido práctico. Buenos Aires: Siglo XXI.

(2011a): «Los tres estados del capital cultural». En Las estrategias de reproducción social. Buenos Aires: Siglo XXI.

(2011b): «Estrategias de reproducción y modos de dominación». En Las estrategias de reproducción social. Buenos Aires: Siglo XXI.

— y JEAN- ClAUdE PASSERON (1998): La reproducción. Elementos para una teoría del sistema de enseñanza. México: Fontamara.

CAFIER, MAGDALENA (2007): «La escuela en la vida cotidiana de familias en contextos de pobreza». En AMALIA EgUía y SUSANA ORTALE: Los significados de la pobreza. Buenos Aires: Biblos.

GRIGNON, ClaUde Y JEAN- ClAUde PASSERON (1991): Lo culto y lo popular. Miserabilismo y populismo en sociología y literatura. Buenos Aires: Nueva Visión.

GuTIÉRREZ, AliCIA (2005): Pobre como siempre. Estrategias de reproducción social en la pobreza. Córdoba: Ferreyra Editor.

(2012): Las prácticas sociales. Una introducción a Pierre Bourdieu. Villa María: EDUVIM.

— y HÉCTOR MANSILLA (2013): «El espacio social y su reproducción: aspectos teórico metodológicos y fuentes secundarias». Ponencia expuesta en el XXIX Congreso ALAS, Santiago.

JACINTO, ClAUdia y VERÓNICA MiLlANAAR (2009): «Enfoques de programas para la inclusión laboral de los jóvenes pobres: lo institucional como soporte subjetivo». Última Década №30. Valparaíso: Ediciones CIDPA.

— «Los nuevos saberes para la inserción laboral. Formación para el trabajo con jóvenes vulnerables en Argentina». Revista Mexicana de Investigación Educativa, Vol. 17, №52.

LAMONT, Michele y ANNETTE LAREAU (1988): «Cultural Capital: Allusions, Gaps and Glissandos in Recent Theoretical Developments». Sociological Theory, Vol. 6, №2. American Sociological Association.

KESSLER, GABRIEL (2000): «Redefinición del mundo social en tiempos de cambio. Una tipología para la experiencia del empobrecimiento». En M. Svampa (editora): Desde abajo. La transformación de las identidades sociales. Buenos Aires: Biblos.

MíGUEZ, DANIEL y AlEJANDRO ISAL (2010): Entre la inseguridad y el temor. Instantáneas de la sociedad actual. Buenos Aires: Paidós.

y PABLO SEMÁN (2006): «Diversidad y recurrencia en las culturas populares actuales». En D. MíGUEZ y P. SEMÁN (editores): Entre santos, cumbias y piquetes. Las culturas populares en la Argentina reciente. Buenos Aires: Biblos. 
Murmis, Miguel y SiLVio FeldMAN (1993): «La heterogeneidad social de las pobrezas». En A. MINUJIN: Cuesta abajo. Los nuevos pobres: efectos de la crisis en la sociedad argentina. Buenos Aires: UNICEF/LOSADA.

NAROdowski, PATRICIO, DEMIAN PANIGO y Nicolás DVOSKIN (2010): «Aspectos teóricos relevantes para el análisis empírico de la informalidad en la Argentina». En J. C. NeFFA, D. PANIGO y P. E. PÉREZ: Transformaciones del empleo en Argentina. Estructura, dinámica e instituciones. Buenos Aires: CICCUS.

NefFA, Julio C., BRENDA BROWn y EMILIANO LóPEZ (2012): «Empleo, desempleo y políticas de empleo. Políticas activas de empleo durante la postconvertibilidad». №11, Tercer Trimestre. Buenos Aires: CEIL-PIETTE.

- - MARÍA L. OlIVERI y JULIANA PERSIA BROWN (2010): «Transformaciones del mercado de trabajo en la Argentina: 1974-2009». En J. C. NefFA, D. PANigo y P. E. PÉREZ: Transformaciones del empleo en Argentina. Estructura, dinámica e instituciones. Buenos Aires: CICCUS.

PÉREZ, PABLO (2013): «Inserción laboral juvenil y políticas públicas de empleo en Argentina». Revista Juventude.br. Centro de Estudos e Memória da Juventude.

Salvia, Agustín, Luciana Fragulia y Úrsula Metlika (2006): «¿Disipación del desempleo o espejismos de la Argentina post devaluación?». Lavboratorio, Año 8, Nº.

SVAmPA, MARISTElla (2005): La sociedad excluyente. La Argentina bajo el signo del neoliberalismo. Buenos Aires: Taurus.

VARGAS, PATRICIA (2005): Bolivianos, paraguayos y argentinos en la obra: identidades étnico-nacionales entre los trabajadores de la construcción. Buenos Aires: IDES-Antropofagia.

WELLER, JURGEN (2008): «Oportunidades y obstáculos. Las características de la inserción laboral juvenil en economías en expansión». Revista del Trabajo (Nueva época), Año $4, \mathrm{~N}^{\circ} 6$. Buenos Aires.

Willis, Paul (1988): Aprendiendo a trabajar. Cómo los chicos de clase obrera consiguen trabajos de clase obrera. Madrid: Akal. 